\title{
A method for incorporating organ motion due to breathing into 3D dose calculations
}

\author{
Anthony E. Lujan, ${ }^{\text {a) }}$ Edward W. Larsen, James M. Balter, and Randall K. Ten Haken \\ Department of Nuclear Engineering and Radiological Sciences, Department of Radiation Oncology, \\ University of Michigan, Ann Arbor, Michigan 48109
}

(Received 31 July 1998; accepted for publication 24 February 1999)

\begin{abstract}
A method is proposed that incorporates the effects of intratreatment organ motion due to breathing on the dose calculations for the treatment of liver disease. Our method is based on the convolution of a static dose distribution with a probability distribution function (PDF) which describes the nature of the motion. The organ motion due to breathing is assumed here to be one-dimensional (in the superior-inferior direction), and is modeled using a periodic but asymmetric function (more time spent at exhale versus inhale). The dose distribution calculated using convolution-based methods is compared to the static dose distribution using dose difference displays and the effective volume $\left(V_{\text {eff }}\right)$ of the uninvolved liver, as per a liver dose escalation protocol in use at our institution. The convolution-based calculation is also compared to direct simulations that model individual fractions of a treatment. Analysis shows that incorporation of the organ motion could lead to changes in the dose prescribed for a treatment based on the $V_{\text {eff }}$ of the uninvolved liver. Comparison of convolution-based calculations and direct simulation of various worst-case scenarios indicates that a single convolution-based calculation is sufficient to predict the dose distribution for the example treatment plan given. (C) 1999 American Association of Physicists in Medicine.
\end{abstract}

[S0094-2405(99)00405-8]

Key words: organ motion, uncertainty, treatment verification, treatment planning, dose calculations

\section{INTRODUCTION}

Uncertainties in the target position in conformal radiotherapy arise from daily setup uncertainties and organ motion. Setup uncertainties can be reduced via immobilization techniques and careful daily repositioning of the patient using portal imaging and alignment tools. ${ }^{1,2}$ For treatment sites in the abdomen, intratreatment organ motion occurs due to breathing, and efforts are underway to minimize the resulting position uncertainties. ${ }^{3-10}$ Two primary approaches exist to account for the resulting uncertainties in the planned dose distribution. The "traditional" approach measures or estimates the extent of setup uncertainty and organ motion and adds margins around a clinical tumor volume (CTV) to form a planning target volume (PTV). The dose is calculated on a static patient model and prescribed to the PTV with the intent that the CTV will almost always lie within the area defined by the PTV. However, this margin expansion approach does not describe the effects of the uncertainties on the normal tissues near the CTV. The second approach also includes margins for error but incorporates the uncertainties into the dose calculations, thereby giving more complete and accurate information on the dose delivered to both the target volume and the nearby normal tissues.

Methods based on a convolution of the static dose distribution with a function (generally Gaussian) representing the distribution of uncertainties from setup and intertreatment organ motion have been proposed for sites in the pelvis. ${ }^{11-15}$ The goal of the present work is to extend convolution-based methods to incorporate uncertainties from intratreatment or- gan motion due to breathing into 3D dose calculations. In this paper, we confirm the validity of this approach via comparisons to direct simulations for treatment of tumors in the liver. Also, we retrospectively analyze the effects of the uncertainties on the treatment plan and dose prescription with a treatment protocol for liver disease used at the University of Michigan. ${ }^{16-18}$

\section{METHODS AND MATERIALS}

The basic algorithm for convolving setup uncertainties with a static dose distribution has been described previously. ${ }^{11-15}$ We have extended the general convolution of setup errors to include the effects of organ motion due to breathing. Use of the convolution method assumes rigid body motion. Several authors ${ }^{19-21}$ have noted that the motion due to breathing of some organs in the abdomen, particularly the liver, is predominantly (though not exclusively) in the superior-inferior (SI) direction. This general convention is supported by computed tomography (CT) studies. ${ }^{3}$ Thus, we have assumed for this study that the organ motion is onedimensional along the SI axis. This motion can be incorporated into a dose distribution using a convolution, as described in Eq. (1). (Although limited to one-dimensional motion here for clarity, the formalism is readily applicable for more complete rigid body motion using $\bar{D}\left(\mathbf{r}^{\prime}\right)$ $\left.=\int D_{0}\left(\mathbf{r}^{\prime}-\mathbf{r}^{\prime}\right) p_{\text {om }}\left(\mathbf{r}^{\prime}\right) d \mathbf{r}^{\prime}.\right)$

$$
\bar{D}(x, y, z)=\int D_{0}\left(x, y, z-z^{\prime}\right) p_{o m}\left(z^{\prime}\right) d z^{\prime},
$$




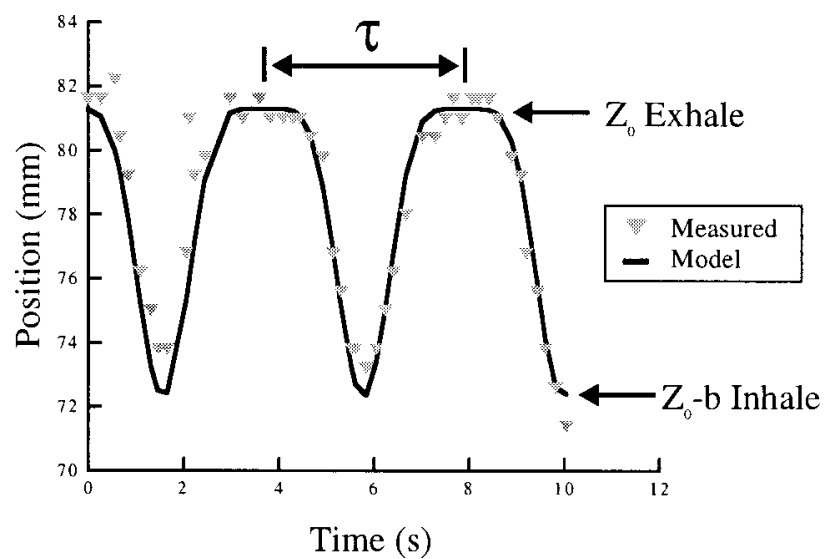

FIG. 1. Position of diaphragm due to breathing seen in fluoroscopic studies (triangles) and mathematical model of data (solid line).

where $\bar{D}(x, y, z)$ is the modified dose distribution at a point $x, y, z, D_{0}\left(x, y, z-z^{\prime}\right)$ is the original dose distribution at a point $x, y, z-z^{\prime}$, and $p_{o m}\left(z^{\prime}\right)$ represents the probability distribution function describing the organ motion due to breathing along the superior-inferior (SI) axis (details below).

The form of $p_{o m}(z)$ was found by considering the nature of the organ motion due to breathing. Unlike setup uncertainties, the organ motion due to breathing is an intratreatment motion. Davies ${ }^{21}$ found that the motion of the liver is well correlated with the motion of the diaphragm. Balter et al., observed under fluoroscopy that the motion of the diaphragm due to breathing is generally periodic but asymmetric, with the majority of time spent at the exhale position, as shown in Fig. $1 .{ }^{22}$ These results are similar to those reported by Kubo. ${ }^{6}$ Other studies have shown, however, that the period and amplitude of the motion due to breathing can vary, even over a short period of time. ${ }^{7,23}$ Our current model assumes a fixed period and amplitude of motion, where the values for the period and amplitude are selected to represent an average observed amplitude and period (Fig. 1).

Assuming a fixed period for the motion, the position of the organ as a function of time can be parameterized. A mathematical model that describes this type of motion is

$$
z(t)=z_{0}-b \cos ^{2 n}(\pi t / \tau-\phi),
$$

where $z_{0}$ is the position at exhale, $b$ is the extent (amplitude) of the motion, $z_{0}-b$ is the position at inhale, $\tau$ is the period of breathing cycle, $n$ is a parameter that determines general shape (steepness and flatness) of the model, and $\phi$ is the starting phase of the breathing cycle. For the example case shown, we used a period for the breathing cycle $\tau=4.2 \mathrm{~s}$, an extent of motion $b=1.5 \mathrm{~cm}$, and a value of $n=3$.

Patients on the liver treatment protocol at the University of Michigan (UM) were CT-scanned at the exhale position. ${ }^{22}$ We used the relationship in Eq. (2) along with the following definitions to generate a probability distribution function (PDF) for points in the liver being a distance, $z$, away from the starting position at exhale (for $\frac{1}{2}$ a breathing cycle). $p_{o m}(z) d z$ is the probability that a point lies between $z$ and $z+d z$ and is equal to the fraction of the total time that a point spends in the interval between $t$ and $t+d t$

$$
\begin{aligned}
& p_{\text {om }}(z) d z=\frac{d t}{\tau / 2}, \\
& p_{\text {om }}(z)=\frac{2}{\tau} \frac{d t}{d z} .
\end{aligned}
$$

We wish to express the PDF as a function of position rather than a function of time, so Eq. (2) is solved for $t$ and used in Eq. (3), yielding the following expression:

$$
\begin{aligned}
p_{\text {om }}(z)= & \left\{n b \pi\left(\frac{z_{0}-z}{b}\right)^{(2 n-1) / 2 n}\right. \\
& \left.\times\left[1-\left(\frac{z_{0}-z}{b}\right)^{1 / n}\right]^{1 / 2}\right\}^{-1} \text { for } z_{0}-b<z<z_{0} .
\end{aligned}
$$

This PDF integrates to 1 over the interval $z_{0}-b<z$ $<z_{0}$, as desired (and required for a probability distribution function), and is a function of the extent of motion $b$, and the shaping parameter $n$, but not the beam-on time $T$ or the starting phase $\phi$.

Use of the PDF described in Eq. (4) (integrated over the extent of motion from $z_{0}-b$ to $z_{0}$ ) in the convolution [Eq. (1)] determines the dose distribution that would result from a patient receiving an infinite number of fractions and being treated over an infinite number of breathing cycles per fraction. In reality, the patient is treated for a finite number of fractions and a finite number of breathing cycles. The PDFs representing the nature of motion for the same treatment time $T$ but different starting phases $\phi$ differ from each other and from the PDF generated using Eq. (4). A direct simulation $^{15,24}$ of each situation would more accurately account for the organ motion due to breathing. However, to reflect an entire course of treatment consisting of many individual fractions, a large number of simulations would need to be performed. Hence, a single convolution calculation would often be preferred in treatment planning applications. We performed direct simulations of the breathing cycle for individual treatments to determine how neglecting the starting phase $\phi$ and the treatment time $T$ might affect the convolution calculation. Our procedure is described below.

1. As shown in Fig. 2, we discretized the organ position as a function of time into 10 bins. The organ position in each bin was computed from the time-weighted average of the organ position in that bin.

2. The change in organ position was simulated in the treatment planning system by moving the beams and isocenter in a direction opposite the motion [i.e., only in the $z$ (SI) direction].

3. The dose distribution was recalculated for each simulated position.

4. We computed the probability $p_{b i}$ of finding the organ in the $i$ th bin based on the mathematical model of the organ motion given in Eq. (2). 


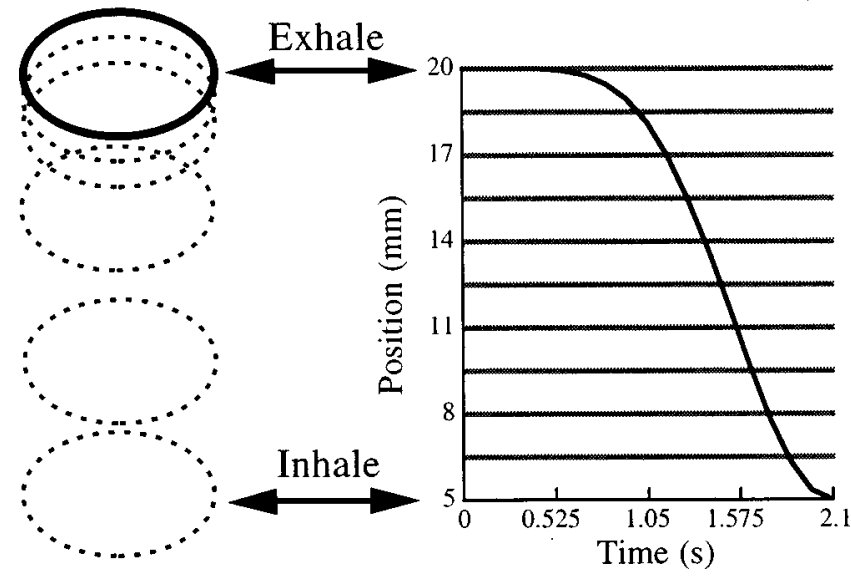

FIG. 2. Organ position as a function of time discretized into 10 bins for one half of a breathing cycle (exhale to inhale).

5. Each calculation at a simulated position was weighted by $p_{b i}$.

6. Beam weights were adjusted by the ratio of tissue phantom ratios to account for changes in SSD and calculation depth due to changes in the external contour.

7. Individual simulations were summed to form a composite dose distribution using the treatment planning geometry as a backdrop.

The calculated probability $p_{b i}$ of the organ residing in bin $i$ is a function of both the starting phase, $\phi$ (point in breathing cycle when the treatment begins) and the treatment time, $T$ (beam-on time), $p_{b i}=p_{b i}(\phi, T)$. We computed $p_{b i}$ for onehalf of a breathing cycle (exhale to inhale, $\phi=0$ ) and will henceforth refer to this probability as the global or standard probability per bin, $p_{g i}$. This is a discrete version of the PDF that we can calculate using Eq. (4), which assumes that the starting phase does not affect the probability of an organ moving into a given position (i.e., the treatment beam-on time is equal to an integral number of symmetric half-cycles of the breathing).

We calculated $p_{b i}(\phi, T)$ for treatment times ranging between 4 and 9 breathing cycles $(\sim 8-20 \mathrm{~s})$. For each $T$, we varied $\phi$ over one complete breathing cycle (from exhale to exhale) and compared $p_{b i}(\phi, T)$ to the global probabilities $p_{g i}$ to determine how the probabilities varied as a function of $\phi$ and $T$.

For each beam in a treatment, $\sim 100$ monitor units (M.U.) are delivered at a rate of $\sim 300 \mathrm{M} . \mathrm{U} . / \mathrm{min}$. This leads to beam-on times of $\sim 20 \mathrm{~s}$. We specifically examined a treatment time of $T=T_{s}=9.45 \mathrm{~s}$ (2.25 breathing cycles) for our worst case simulations. This treatment time is shorter than most of our expected treatment times but is on the correct time scale for segmented radiation therapy treatment at UM, which is delivered at $600 \mathrm{M} . \mathrm{U} . / \mathrm{min}$ ( $\sim 10 \mathrm{~s}$ beam-on time). Thus, we calculated $p_{b i}\left(\phi, T_{s}\right)$ and determined the starting phases, $\phi_{j}(j=1-10)$, that resulted in maximum differences for each of the 10 bins.

We simulated the organ motion for each of those starting phases, $\phi_{j}$, using the appropriate probability distribution, $p_{b i}\left(\phi_{j}, T_{s}\right)$ in the direct simulation. These simulations rep- resent potential (but highly unlikely) worst case scenarios, in which each treatment would begin at the same starting phase $\phi_{j}$, and last for the same treatment time $T_{s}$, thus each treatment fraction is synchronized to yield a probability distribution $p_{b i}\left(\phi_{j}, T_{s}\right)$ that is most different from the global probability used for the convolution.

For each of our simulations, we generated dose volume histograms (DVHs) for the CTV and the uninvolved liver. From the DVH, we calculated the effective volume, $V_{\text {eff }},{ }^{25-27}$ for the uninvolved liver. We compared DVHs and $V_{\text {eff }}$ values for all generated dose distributions.

The treatment planning geometry used for the simulation presented here is shown in Fig. 3(a). The gross tumor volume (GTV) was located in the anterior-inferior region of the liver. The resultant PTV was treated using a pair of axial beams [RPO (right-posterior-oblique), LPO (left-posterioroblique)] complimented by a wedged oblique off-axis beam [right-anterior-superior-oblique (RASO)]. The PTV was completely covered by the $95 \%$ isodose surface.

For a reference simulation, we compared the dose distribution calculated via the convolution method [Eq. (1)] to the static (initial treatment plan) dose distribution. Next, we compared the convolved dose distribution to the dose distribution calculated via direct simulation using the global PDF. We then found the difference between the dose distribution calculated using the global PDF and the dose distributions calculated from the worst case scenarios.

\section{RESULTS}

A dose difference display between the convolved dose distribution and the static dose distribution is shown in Fig. 3(b). For this particular treatment plan and tumor location (recall, CT scan was obtained at exhale), we could expect the motion due to breathing to cause regions superior to the superior beam edges to move into the beam and regions superior to the inferior beam edges to move out of the beam [observed by comparison of Figs. 3(a) and 3(b)]. Differences up to $26 \%$ are calculated between the convolved dose distribution and the static dose distribution in regions outside of the CTV.

The dose distribution including motion due to breathing shows that the planned dose is delivered to the CTV, indicating that the margins chosen for the PTV were sufficient. Examination of the DVH of the uninvolved liver agree with our observations for this plan. For this tumor location and treatment plan, organ motion will result in an increase in the mean dose delivered to normal liver compared with the dose calculated for the static treatment plan. This also translates into an increase in $V_{\text {eff }}$ for the convolved dose distribution, relative to the static plan, sufficient to suggest a change in the prescription dose assigned for this treatment plan to maintain a fixed level of toxicity. ${ }^{18,26}$

Figure 4 shows the probability of being in bin $10, p_{b 10}$, for three different treatment times. As can be seen, the probabilities for bin 10 vary periodically about the global probability (as expected, based on the nature of the motion itself) as the starting phase, $\phi$ varies across the breathing cycle. In 

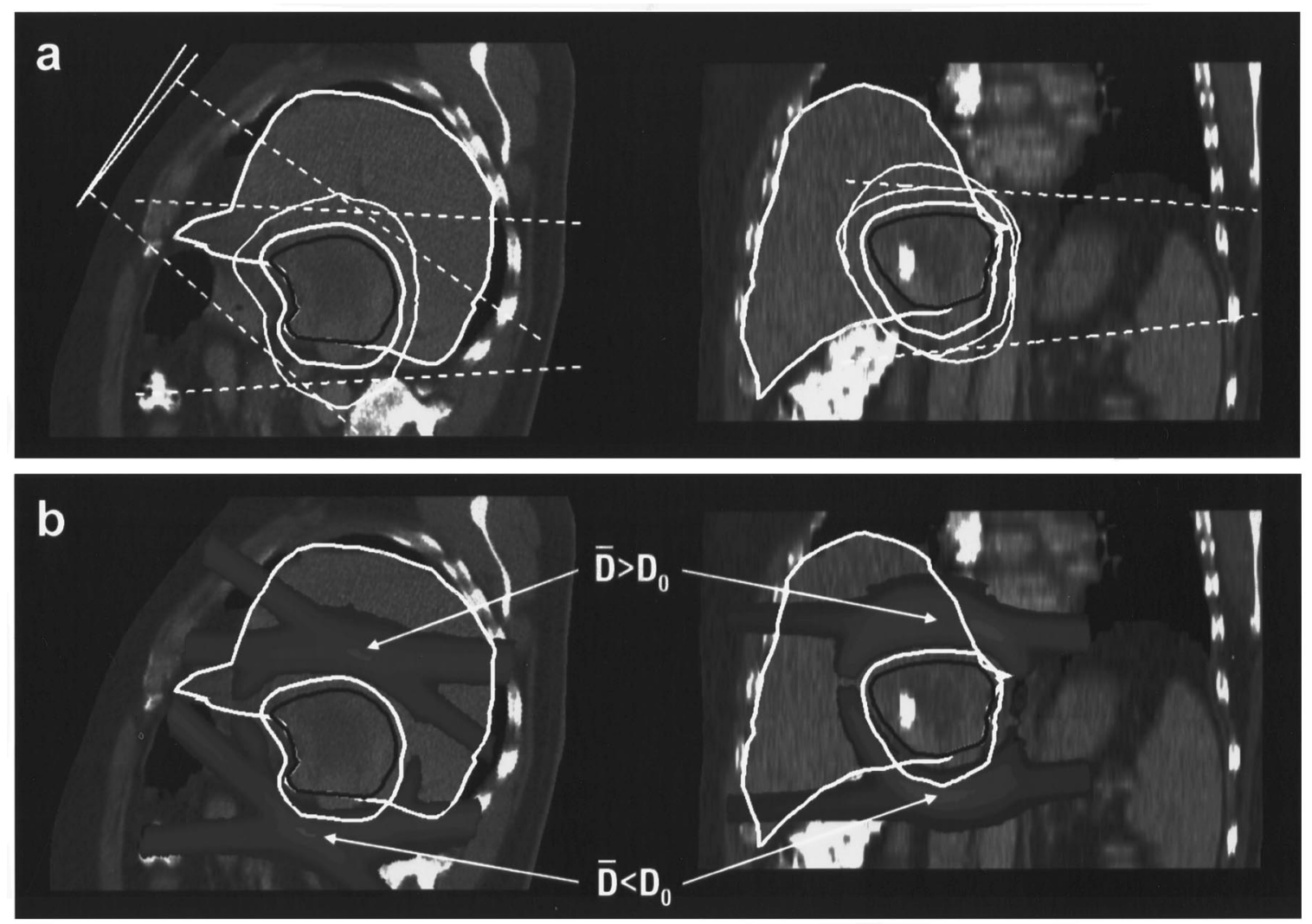

FIG. 3. (a) Example three-field liver tumor treatment plan comprising a pair of axial beams, right-posterior-oblique (RPO) and left-posterior-oblique (LPO), and a wedged oblique off-axis beam, right-anterior-superior-oblique (RASO). PTV volume includes a superior margin of $0.3 \mathrm{~cm}$ and an inferior margin of 1.5 $\mathrm{cm}$ for breathing as well as a uniform $0.5 \mathrm{~cm}$ margin for setup uncertainties, all with respect to the CTV (black contour). (b) Dose difference display between convolved dose distribution (including organ motion) and static dose distribution. Regions where the convolved dose distribution receives more dose than the static dose distribution would predict are indicated as $\bar{D}>D_{0}$. Regions where the convolved dose distribution receives less dose than predicted by the static dose distribution are indicated as $\bar{D}<D_{0}$.

addition, as the treatment time $T$ increases, the maximum differences between $p_{b i}$ and $p_{g i}$ decrease. Cumulative DVHs for the uninvolved liver generated from the dose distributions calculated using the global probability distribution and

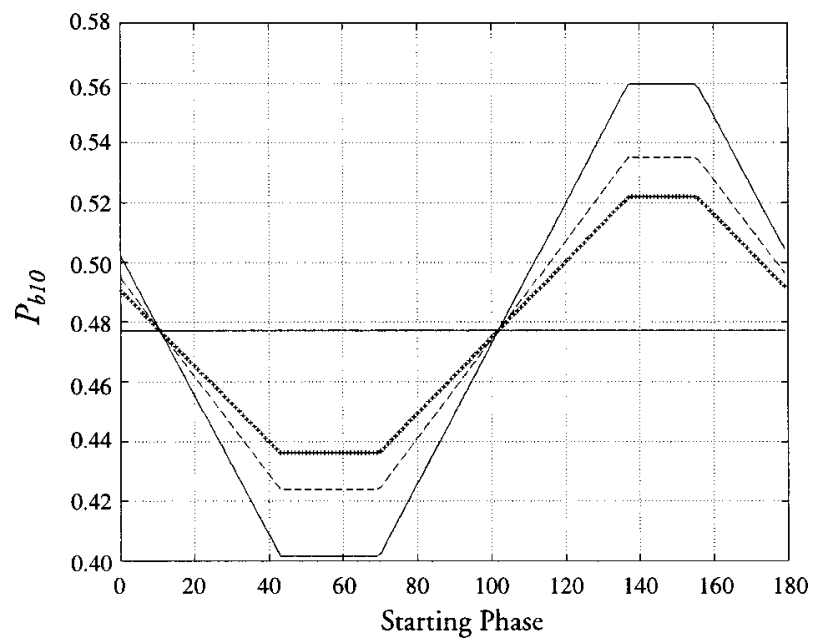

FIG. 4. $p_{b 10}$ for three treatment times, $T$, as a function of the starting phase, $\phi$. Solid line: $T_{1}=9.975 \mathrm{~s}$, dashed line: $T_{2}=14.175 \mathrm{~s}$, hatched line: $T_{3}$ $=18.357 \mathrm{~s}$, horizontal line: $p_{g 10}=0.477$. the worst case scenario PDFs were nearly identical for each scenario. The dose distributions themselves agreed to within $\pm 2 \%$ for $\sim 98 \%$ of the calculation volume (for a single worst case simulation). The $V_{\text {eff }}$ values calculated for the worst case scenarios differed from the $V_{\text {eff }}$ from the global PDF simulation by less than $0.35 \%$; quite insufficient to cause consideration of changes in prescription dose.

The dose distribution calculated using the convolutionbased method and the dose distribution calculated via a direct simulation using the global PDF were nearly identical, with dose differences of less than $\pm 2 \%$ to over $98 \%$ of the uninvolved liver volume. The $V_{\text {eff }}$ values calculated via each method differed insignificantly $(\approx 0.2 \%)$.

\section{DISCUSSION}

Intratreatment organ motion due to breathing can lead to erroneous prediction of the dose delivered to a patient when a static computed tomography (CT) scan of a patient is used for treatment planning dose calculations. Though the example shown is specific to a particular treatment planning geometry, the importance of incorporating the organ motion due to breathing in the dose calculation is clear. That is, 
including the effects of breathing on the calculation of dose to these normal tissues (liver) can lead to changes in prescription dose in our protocol setting.

We have also compared direct simulations using a global PDF to the convolution calculation and found good agreement between the dose distributions generated via the two procedures. This was expected, because the global PDF is a discrete version of the PDF used in the convolution calculation.

This convolution-based procedure and the direct simulation using the global PDF yield dose distributions resulting from the patient receiving an infinite number of fractions, or equivalently, being treated over an infinite number of breathing cycles. The PDF generated for the convolution calculation neglects the effects of the finite beam-on time and the starting phase of the breathing cycle. However, we hypothesized that the phase differences would cancel out in a simulation of a treatment because there are sufficiently many fractions for each treatment. This was verified by considering potential worst case scenarios in the simulations. We can expect that the starting phase, unless monitored, will vary randomly for each fraction. Hence, the differences for each bin will cancel out. This means that we can simulate the organ motion due to breathing using our simple global probability in a direct simulation or convolution-based calculation and do not need to simulate each fraction to model the organ motion due to breathing.

As noted above, studies have shown that the period and amplitude of the motion due to breathing can vary. ${ }^{7,23}$ Our current model assumes a fixed period and amplitude of motion chosen to represent an average observed amplitude and period, and thus an average expected dose distribution including the effects of breathing. As with the starting phase of breathing, the unexpected variations in period and amplitude should average out over the course of a treatment.

The convolution calculation is applied to the entire dose grid. However, some structures in the grid (e.g., the spinal cord) may not move according to the observations in Fig. 1. The dose to such structures must be analyzed using other methods or different PDFs as the simulated dose distribution calculated using the above procedure only represents dose to structures that move according to those observations.

Our results are specific for the patient geometry and treatment plan shown. However, for standard axial treatment plans in the abdomen, we would expect the general results to apply (i.e., regions superior to the superior beam edges move into the beam and regions superior to the inferior beam edges will move out of the beam). The specific magnitude of the changes will depend on the actual treatment plan.

The convolution-based procedure given by Eq. (1) will apply in regions where the dose distribution is invariant under small changes in the setup orientation. In regions where this is not true (such as the lung-tissue interface and the surface-patient interface), careful direct simulation of the motion will provide more accurate computation of the average dose distribution including uncertainties. Also, rigid body motion assumes no change in the patient external contour and no organ deformation. Patient breathing can result in small (typically $<1 \mathrm{~cm}$ ) changes in beam pathlength. These changes can lead to small changes in dose for single beams. ${ }^{3,21}$

Further, as the nature of the organ motion is better understood and parameterized, confirmation of a convolutionbased procedure such as in Eq. (1), will require a more complete direct simulation which includes the effects of changes in the patient external contour and any organ deformation which may occur. In addition, a more complex PDF may be required to model the more complex motion. We have presented a basic framework from which additional study may occur.

\section{ACKNOWLEDGMENTS}

Work supported by NIH Grant No. P01-CA59827. Dr. Balter is supported as a Kimmel Scholar.

${ }^{a}$ Electronic mail: aelujan@umich.edu

${ }^{1}$ K. G. A. Gilhuijs, K. Drukker, A. Touw, P. J. van de Ven, and M. van Herk, "Interactive three dimensional inspection of patient setup in radiation therapy using digital portal images and computed tomography data,', Int. J. Radiat. Oncol., Biol., Phys. 34, 873-885 (1996).

${ }^{2}$ A. E. Lujan, J. M. Balter, and R. K. Ten Haken, "'Determination of rotations in three dimensions using two dimensional portal image registration,' Med. Phys. 25, 703-708 (1998).

${ }^{3}$ J. M. Balter, R. K. Ten Haken, T. S. Lawrence, K. L. Lam, and J. M. Robertson, "Uncertainties in CT-based radiation therapy treatment planning associated with patient breathing,', Int. J. Radiat. Oncol., Biol., Phys. 36, 167-174 (1996).

${ }^{4}$ S. C. Davies, A. L. Hill, R. B. Holmes, M. Halliwell, and P. C. Jackson, "Ultrasound quantitation of respiratory organ motion in the upper abdomen,’ Br. J. Radiol. 67, 1096-1102 (1994).

${ }^{5}$ J. Hanley, M. M. Debois, A. Raben, G. S. Mageras, W. R. Lutz, B. Mychalczak, L. H. Schwartz, P. J. Gloeggler, S. A. Leibel, Z. Fuks, and G. J. Kutcher, "Deep inspiration breath-hold technique for lung tumors: the potential value of target immobilization and reduced lung density in dose escalation,', Int. J. Radiat. Oncol., Biol., Phys. 36, Supplement, 188 (1996).

${ }^{6}$ H. D. Kubo and B. C. Hill, "'Respiration gated radiotherapy: a technical study,'” Phys. Med. Biol. 41, 83-91 (1996).

${ }^{7}$ C. J. Ritchie, J. Hsieh, M. F. Gard, J. D. Godwin, Y. Kim, and C. R. Crawford, "Predictive respiratory gating: a new method to reduce motion artifacts on CT scans,', Radiology 190, 847-852 (1994).

${ }^{8}$ S. M. Morrill, M. Langer, and R. G. Lane, "Real-time couch compensation for intratreatment organ motion: Theoretical advantages," Med. Phys. 23, 1083 (1996).

${ }^{9}$ R. K. Ten Haken, J. M. Balter, L. H. Marsh, J. M. Robertson, and T. S. Lawrence, "Potential benefits of eliminating planning target volume expansions for patient breathing in the treatment of liver tumors," Int. J. Radiat. Oncol., Biol., Phys. 38, 613-617 (1997).

${ }^{10}$ J. W. Wong, M. B. Sharpe, and D. A. Jaffray, "Minimizing intra-fraction organ motion with active breathing control," Med. Phys. 24, 1022 (1997).

${ }^{11}$ A. Bel, M. van Herk, and J. M. Lebesque, "Target margins for random geometrical treatment uncertainties in conformal radiotherapy,' Med. Phys. 23, 1537-1545 (1996).

${ }^{12}$ C. S. Chui, G. Kutcher, and T. LoSasso, "A convolution method for incorporating uncertainties in dose calculations,', Med. Phys. 19, 814 (1992).

${ }^{13}$ M. A. Hunt, T. E. Schulttheiss, G. Desobry, and G. E. Hanks, "Convolving setup uncertainties with dose distributions,' Med. Phys. 20, 929 (1993).

${ }^{14}$ G. J. Kutcher, C. Chui, and T. LoSasso, “Incorporation of set-up uncertainties in treatment planning calculations,', Int. J. Radiat. Oncol., Biol., Phys. 21, 123 (1991).

${ }^{15}$ J. Leong, "Implementation of random positioning error in computerized radiation treatment planning systems as a result of fractionation,' Phys. Med. Biol. 32, 327-334 (1987). 
${ }^{16}$ T. S. Lawrence, R. J. Tesser, and R. K. Ten Haken, “An application of dose volume histograms to the treatment of intrahepatic malignancies with radiation therapy," Int. J. Radiat. Oncol., Biol., Phys. 19, 10411047 (1990).

${ }^{17}$ T. S. Lawrence, R. K. Ten Haken, M. L. Kessler, J. M. Roberston, J. T. Lyman, M. L. Lavigne, M. B. Brown, D. J. DuRoss, J. C. Andrews, W. D. Ensminger, and A. S. Lichter, "The use of 3D dose volume analysis to predict radiation hepatitis,' Int. J. Radiat. Oncol., Biol., Phys. 23, 781788 (1992).

${ }^{18}$ C. J. McGinn, R. K. Ten Haken, W. D. Ensminger, S. Walker, S. Wang, and T. S. Lawrence, "The treatment of intraheptatic cancers with radiation doses based on a normal tissue complication probability model,' J. Clin. Oncol. 16, 2246-2252 (1998).

${ }^{19}$ I. Suramo, M. Päivänsalo, and V. Myllylä, "Cranio-caudal movements of the liver, pancreas and kidneys in respiration," Acta Radiol.: Diagn. 25, 129-131 (1984).

${ }^{20}$ H. W. Korin, R. L. Ehman, S. J. Riederer, J. P. Felmlee, and R. C. Grimm, "Respiratory Kinematics of the upper abdominal organs: A quantitative study,', Magn. Reson. Med. 23, 172-178 (1992).

${ }^{21}$ S. C. Davies, A. L. Hill, R. B. Holmes, M. Halliwell, and P. C. Jackson, "Ultrasound quantitation of respiratory organ motion in the upper abdomen,' Br. J. Radiol. 67, 1096-1102 (1994).

${ }^{22}$ J. M. Balter, K. L. Lam, C. J. McGinn, T. S. Lawrence, and R. K. Ten
Haken, "Improvement of CT-based treatment planning models of abdominal targets using static exhale imaging,', Int. J. Radiat. Oncol., Biol., Phys. 41, 939-943 (1998).

${ }^{23}$ M. R. Sontag, Z. W. Lai, B. W. McRoy, and R. D. Waters, "Characterization of respiratory motion for pediatric conformal 3-D Therapy,' Med. Phys. 23, 1082 (1996).

${ }^{24}$ J. H. Killoran, H. M. Kooy, D. J. Gladstone, F. J. Welte, and C. J. Beard, "A numerical simulation of organ motion and daily setup uncertainties: Implications for radiation therapy,' ' Int. J. Radiat. Oncol., Biol., Phys. 37, 213-221 (1997).

${ }^{25}$ G. J. Kutcher and C. Burman, "Calculation of complication probability factors for non-uniform normal tissue irradiation: The effective volume method,' Int. J. Radiat. Oncol., Biol., Phys. 16, 1623-1630 (1989).

${ }^{26}$ R. K. Ten Haken, M. K. Martel, M. L. Kessler, M. B. Hazuka, T. S. Lawrence, J. M. Roberston, A. T. Turrisi, and A. S. Lichter, "Use of $V_{\text {eff }}$ and iso-NTCP in the implementation of dose escalation protocols,' Int. J. Radiat. Oncol., Biol., Phys. 27, 689-695 (1993).

${ }^{27}$ G. J. Kutcher and C. Burman, L. Brewster, M. Goitein, and R. Mohan, "Histogram reduction method for calculating complication probabilities for three-dimensional treatment planning evaluations,' Int. J. Radiat. Oncol., Biol., Phys. 21, 137-146 (1991). 